\title{
Políticas linguísticas de formação e de investigação em universidades públicas portuguesas: das tensões a eixos de reflexão-ação
}

\author{
Susana Pinto \& Maria Helena Araújo e Sá
}

Resumo:

Este artigo foca um estudo com universidades públicas portuguesas com o objetivo de identificar e discutir crenças de responsáveis institucionais sobre: (i) o ensino e aprendizagem de línguas no ES; (ii) a utilização das línguas na investigação científica; (iii) o desenvolvimento de políticas linguísticas institucionais de educação e de investigação. Atores institucionais responsáveis pelas estratégias pedagógicas e pelas atividades de investigação em oito universidades públicas portuguesas responderam a um inquérito por questionário e participaram em entrevistas semiestruturadas. Os resultados evidenciam tendências comuns nas universidades e apontam para uma tensão entre, por um lado, a existência de práticas institucionais que se enquadram em políticas linguísticas de formação e de investigação apoiadas na quase exclusiva utilização da língua inglesa e, por outro lado, a valorização da diversificação do ensino-aprendizagem de línguas no ensino superior e a necessidade de uma ciência mais plurilingue que privilegie o português como língua de ciência. Os resultados permitem delinear um conjunto de linhas de reflexão-ação dirigidas às instituições de ensino superior portuguesas, visando o desenvolvimento de políticas linguísticas institucionais multilingues.

Palavras-chave:

universidades públicas portuguesas; políticas linguísticas educativas; políticas linguísticas de investigação. 


\title{
Training and research language policies in Portuguese public universities: from tensions to reflection-action axes
}

\begin{abstract}
This paper reports on a study that looked at Portuguese public universities setting out to identify and discuss institutional stakeholders' beliefs concerning: (i) language teaching and learning in HE; (ii) the use of languages in scientific research; (iii) the development of institutional language education policies and language policies within scientific research. Institutional stakeholders responsible for pedagogical strategies and for research activities at eight Portuguese public universities completed a questionnaire and participated in in-depth interviews. The findings indicate there are common tendencies regarding beliefs that point, mainly, to a tension between, on one hand, the existence of reported practices that centre on "English-mainly" education and research language policies and, on the other hand, the valorisation of a diversified language teaching and learning in higher education and the need for a more plurilingual science that privileges Portuguese as a science language. The results allow outlining a set of reflectionaction lines addressed at Portuguese $\mathrm{HEI}$, aiming at the development of multilingual institutional language policies.
\end{abstract}

Keywords: Portuguese public universities; language education policies; research language policies.

\section{Políticas lingüísticas de formación e investigación en universidades públicas portuguesas: de tensiones a líneas de reflexión-acción}

Resumen: Este artículo se centra en un estudio con universidades públicas portuguesas con el objetivo de identificar y discutir creencias de los responsables institucionales sobre: (i) la enseñanza y el aprendizaje de lenguas en la Enseñanza Superior; (ii) la utilización de las lenguas en la investigación científica; (iii) el desarrollo de políticas lingüísticas institucionales de educación y de investigación. Actores institucionales responsables de las estrategias pedagógicas y de las actividades de investigación en ocho universidades públicas portuguesas respondieron a un cuestionario y participaron en entrevistas semiestructuradas. Los resultados evidencian tendencias comunes y apuntan a una tensión entre, por una parte, la existencia de prácticas institucionales que se encuadran en políticas lingüísticas de formación e investigación apoyadas en la casi exclusiva utilización de la lengua inglesa y, por otra, la valoración de la diversificación de la enseñanza y del aprendizaje de idiomas en la enseñanza superior y la necesidad de una ciencia más plurilingüe que privilegie el portugués como lengua científica. Los resultados permiten definir un conjunto de líneas de reflexión-acción destinadas a las universidades portuguesas para el desarrollo de políticas lingüísticas institucionales plurilingües.

Palabras clave: universidades públicas portuguesas; políticas lingüísticas educativas; políticas lingüísticas de investigación.

\section{Politiques linguistiques de formation et de recherche dans des universités publiques portugaises : des tensions aux lignes de réflexion-action}

Résumé: Cet article porte sur une étude menée avec des universités publiques portugaises dans le but d'identifier et de discuter les convictions des dirigeants institutionnels concernant: (i) l'enseignement et l'apprentissage des langues dans l'enseignement supérieur; (ii) l'utilisation des langues dans la recherche scientifique; (iii) l'élaboration de politiques linguistiques institutionnelles pour l'éducation et la recherche. Les acteurs institutionnels responsables des stratégies pédagogiques et des activités de recherche dans huit universités publiques portugaises ont répondu à un questionnaire et participé à des entretiens semi-structurés. Les résultats montrent des tendances communes et font ressortir une tension entre, d'une part, l'existence de pratiques institutionnelles encadrées par les politiques linguistiques de formation et de recherche qui révèlent l'utilisation quasi exclusive de la langue anglaise et, d'autre part, la valorisation de la diversification de l'enseignement-apprentissage des langues dans l'enseignement supérieur et la nécessité d'une science plus plurilingue qui privilégie le portugais comme langue scientifique. Les résultats permettent de délimiter un ensemble de lignes de réflexion-action dirigées aux universités portugaises, visant le développement de politiques linguistiques institutionnelles plurilingues.

Mots-clés: universités publiques portugaises; politiques linguistiques éducatives; politiques linguistiques pour la recherche. 


\section{Políticas linguísticas no ensino superior: a agência dos atores institucionais}

A investigação sobre políticas linguísticas (PL) no ensino superior (ES) tem vindo a receber uma maior atenção, o que não deixa de se relacionar com a importância da sua internacionalização. Neste quadro, nota-se a existência de um crescente interesse na forma como as universidades constroem e desenvolvem PL no âmbito das atividades educativas e investigativas (Liddicoat, 2016).

Em Portugal, esta investigação é ainda reduzida (Pinto, 2012; Pinto \& Araújo e Sá, 2018; Pinto \& Araújo e Sá, em publicação) e o estudo reportado neste texto pretende contribuir para construção de conhecimento na área. O estudo pretendeu mapear e problematizar as tendências das universidades públicas portuguesas quanto à utilização das línguas e ao desenvolvimento de PL na formação e na investigação científica, a partir das vozes de algumas das suas testemunhas privilegiadas: Vice-Reitores para a Formação e para a Investigação. Mais especificamente, o estudo permitiu identificar e discutir práticas reportadas e crenças relativamente: (i) ao ensino-aprendizagem de línguas no ES; (ii) à utilização das línguas na investigação; (iii) ao desenvolvimento de PL institucionais para a formação e investigação.

Crescentemente, a investigação sobre PL no ES tem vindo a focar-se na análise do papel de diversos "micro-level actors" (Baldauf, 2006) enquanto agentes que exercem "individual agency" na construção e implementação de PL institucionais, tais como gestores de governo e de gestão científica e pedagógica (Miranda, Berdugo, \& Tejada, 2016; Siiner, 2016). Os Vice-Reitores para a Formação e Investigação auscultados neste estudo são, de facto, atores institucionais que têm 'the power to influence change' (Zhao \& Baldauf, 2012, p. 3) na medida em que coordenam as estratégias e políticas educativas e investigativas nas suas instituições, podendo influenciar o comportamento de outros atores institucionais no que concerne a PL para a educação e para a investigação.

Considerando a importância da agência destes atores institucionais e reconhecendo que as PL integram três componentes - language practices, language management e language beliefs (Spolsky, 2004) - este artigo foca a componente das language beliefs que dizem respeito ao que indivíduos e sociedades acreditam sobre as línguas e sua utilização, sendo uma manifestação de princípios sociais, culturais e políticos, influenciando discursos e práticas. A relação entre estas language beliefs (que traduzimos como "crenças linguísticas"), as práticas pessoais e societais e as políticas é explicada por Spolsky: “... beliefs both derive from and influence practices. They can be a basis for language management or a management policy can be intended to confirm or modify them" (2004, p. 14).

Tendo em conta o exposto, o artigo estrutura-se da seguinte forma: em primeiro lugar, apresentamos uma visão geral das PL para a educação e investigação no ES 
português; descrevemos depois a metodologia de investigação, seguida dos principais resultados do estudo; por fim, apresentamos as considerações finais e propomos alguns eixos de reflexão-ação dirigidos às universidades portuguesas, no sentido de potenciar $\mathrm{PL}$ institucionais que fomentem um multilinguismo estratégico na formação e na investigação.

\section{Políticas linguísticas de formação e de investigação no ensino superior português}

Em Portugal, macropolíticas linguísticas relativas à formação e investigação no ES são praticamente inexistentes. No que concerne à formação, a utilização das línguas encontra-se presente em escassos Decretos-Lei: o DL N. ${ }^{\circ} 74 / 2006$ que prevê que as IES podem utilizar línguas estrangeiras no ensino em qualquer dos ciclos de estudos, na escrita de dissertações de mestrado, relatórios de estágio de mestrado, trabalhos de projeto e teses de doutoramento, assim como nos atos públicos de defesa; o DL N. ${ }^{\circ} 107 / 2008$ que prevê que os diplomas podem ser plurilingues. Para além destes Decretos-Lei, a questão das línguas na formação apenas pode ser observada no Relatório Uma estratégia para a internacionalização do ensino superior português (MEC, 2014) que defende as necessidades de: "multiplicar a oferta de unidades curriculares em língua inglesa, nos cursos de primeiro ciclo; aumentar o número de mestrados e de doutoramentos em língua inglesa e fomentar a proficiência linguística, abrangendo o inglês e outras línguas necessárias para a internacionalização do sistema" (p. 13). Neste sentido, sublinha-se o facto de o número de cursos em inglês oferecido pelo ES português ser ainda muito baixo, principalmente quando comparado com os países nórdicos, o que se torna um constrangimento à atração de estudantes estrangeiros. Esta ênfase na língua inglesa surge como crucial para o ES e, por isso, recomenda-se "a ampliação da oferta de cursos em língua inglesa e uma melhor preparação linguística dos estudantes portugueses." (p.12). Ao mesmo tempo, sublinha-se a importância do Português na atração de estudantes internacionais, nomeadamente da Comunidade dos Países de Língua Portuguesa (CPLP).

Relativamente à investigação, constata-se que o inglês tem vindo a adquirir o estatuto de língua académica especialmente na publicação (Pinto \& Araújo e Sá, em publicação), o que se prende, claramente, com a premência de uma (restritiva) "internacionalização em inglês", obviamente relacionada com os critérios atuais avaliação da ciência que colocam a ênfase no fator de impacto da produção científica e nos rankings internacionais. Este estatuto de língua potenciadora da internacionalização é sublinhado no Relatório atrás referido:

Naturalmente, na fase que atravessamos, é a língua inglesa que predomina no âmbito do ensino superior e da investigação científica, razão pela qual se 
considera fundamental, por um lado, a proficiência de língua inglesa de todos os estudantes portugueses do ensino superior e, por outro, a multiplicação de formações oferecidas em língua inglesa, em especial os mestrados e doutoramentos (2014, p. 107).

O Relatório também sublinha a importância da língua portuguesa nessa estratégia de internacionalização, língua considerada "instrumento de ciência, de cultura ou de negócio, falada por 250 milhões de pessoas em todo o Mundo" (p.12) e "instrumento de conhecimento e de relação na atividade económica" (p. 91). Esta valorização tinha sido já enfatizada na 2. ${ }^{a}$ Conferência sobre o futuro da língua portuguesa no sistema mundial (Lisboa, outubro 2013) que deu origem ao Plano de Ação de Lisboa (https:// www.cplp.org/Files/Billeder/cplp/1-Resol_PALis.pdf) elaborado pelo Conselho de Ministros da CPLP em 2014. Neste Plano, dão-se recomendações aos membros da CPLP com vista a afirmar o papel da língua portuguesa como língua de ciência, tais como: valorizar o seu uso na comunicação e produção científicas, assim como nos documentos de candidatura e gestão de projetos científicos; promover o desenvolvimento de recursos científicos e de infraestruturas digitais em língua portuguesa, de acesso aberto e gratuito; fomentar medidas para promover a indexação de publicações na SCIELO e na Web of Science.

Em consonância com estas preocupações, a FCT divulgou a Carta de princípios de orientação para a Fundação para a Ciência e a Tecnologia em 2016 (https://www. portugal.gov.pt/media/18501895/20160210-mctes-principios-fct.pdf) onde mostra a intenção de impulsionar "o reforço de comunidades científicas de língua portuguesa e a promoção de indústrias culturais através da língua e do património enquanto veículos de ciência e conhecimento" (p. 3). Apesar disto, não podemos deixar de questionar se este não será um discurso essencialmente retórico já que, por exemplo, o último processo de avaliação das unidades de investigação financiadas pela FCT foi realizado exclusivamente em inglês.

\section{O estudo: objetivos, participantes, recolha e análise de dados}

Este estudo pretendeu identificar as crenças de atores institucionais de universidades públicas portuguesas sobre: (i) o ensino-aprendizagem de línguas no ES; (ii) a utilização das línguas na investigação; (iii) o desenvolvimento de PL institucionais de formação e de investigação. Num primeiro momento, em março de 2014, dois inquéritos por questionário colocados online - um focado na formação/políticas linguísticas educativas e outro focado na investigação/políticas linguísticas investigativas - foram enviados aos Vice-Reitores para a formação e para a investigação das 14 universidades públicas portuguesas. O Quadro 1 sumaria os objetivos de cada um dos questionários: 
Quadro 1. Objetivos dos inquéritos por questionário

\begin{tabular}{|l|l|}
\hline \multicolumn{1}{|c|}{ Inquérito por questionário } & \multicolumn{1}{|c|}{$\begin{array}{c}\text { Inquérito por questionário } \\
\text { educação/políticas linguísticas educativas }\end{array}$} \\
$\begin{array}{l}\text { Identificar crenças e práticas institucionais } \\
\text { relativas ao ensino-aprendizagem de línguas } \\
\text { no ensino superior. }\end{array}$ & $\begin{array}{l}\text { Identificar crenças e práticas institucionais } \\
\text { investigativas } \\
\text { relativas ao estatuto, funções e importância } \\
\text { das línguas na investigação científica. }\end{array}$ \\
$\begin{array}{l}\text { Identificar crenças e iniciativas institucionais } \\
\text { relativas ao desenvolvimento de políticas } \\
\text { linguísticas educativas. }\end{array}$ & $\begin{array}{l}\text { Identificar crenças e iniciativas institucionais } \\
\text { relativas ao desenvolvimento de políticas } \\
\text { linguísticas para a investigação. }\end{array}$ \\
\hline
\end{tabular}

Fonte: autoras

Recebemos respostas de 8 universidades públicas portuguesas, logo 16 inquéritos por questionário (8 relativos à formação e 8 relativos à investigação). A tabela 1 apresenta uma breve caracterização das instituições respondentes.

Tabela 1. Caracterização das universidades participantes

\begin{tabular}{|c|c|c|c|}
\hline Universidade & Ano de criação & $\begin{array}{c}\text { N. }{ }^{\circ} \text { total de } \\
\text { estudantes }\end{array}$ & $\begin{array}{c}\text { N. }{ }^{\circ} \text { de unidades de } \\
\text { investigação }\end{array}$ \\
\hline U1 & 1973 & 13.675 & 18 \\
\hline U2 & 1986 & 6.553 & 18 \\
\hline U3 & 1559 & 8.970 & 11 \\
\hline U4 & 1972 & 8.868 & 8 \\
\hline U5 & 1988 & 3.389 & 8 \\
\hline U6 & 1988 & 5.717 & 7 \\
\hline U7 & 1979 & 9.708 & 41 \\
\hline U8 & 1973 & 20.077 & 4 \\
\hline
\end{tabular}

Após análise de conteúdo das respostas ao inquérito por questionário (Krippendorff, 2013), procedeu-se à realização de inquéritos por entrevista semiestruturada com 11 dos 16 Vice-Reitores para a formação e para a investigação que, inicialmente, preencheram os inquéritos por questionário e que se mostraram disponíveis para a entrevista: 5 Vice-Reitores para a Formação (U1, U2, U6, U7 e U8) e 6 Vice-Reitores para a Investigação (U1, U3, U4, U5, U7 e U6). As entrevistas, gravadas, transcritas e 
sujeitas a análise de conteúdo, foram realizadas com o objetivo de obter maior compreensão das respostas dadas no inquérito por questionário. Os dados recolhidos foram sujeitos a análise de conteúdo categorial no âmbito da qual as categorias de análise surgiram dos próprios dados em interação com os referenciais teóricos relativos a PL de formação e de investigação no ES.

\section{Principais resultados}

De acordo com os objetivos do estudo, os resultados são apresentados em 3 secções: ensino-aprendizagem de línguas; línguas na investigação científica e desenvolvimento de políticas linguísticas institucionais de formação e de investigação. Os dados recolhidos serão analisados de forma holística de modo a permitir uma visão mais integrada dos resultados, destacando-se convergências entre as universidades analisadas.

\section{Ensino-aprendizagem de línguas}

No que diz respeito às práticas institucionais reportadas pelos Vice-Reitores, todos sublinham que o Processo de Bolonha terá tido um impacto negativo no ensino-aprendizagem de línguas nas suas instituições: são oferecidas menos unidades curriculares de línguas e menos cursos as integram por falta de espaço nos currículos. Esta tendência é, também, visível em outras IES europeias, como tem revelado a investigação em diferentes contextos, relacionando-se com a forma como têm vindo a operacionalizar a necessidade de implementar estratégias de internacionalização (Gallagher-Brett \& Broady, 2012). Neste âmbito, a língua inglesa surge como a língua mais importante a ser aprendida no ES através da sua integração em currículos de cursos do $1 .{ }^{\circ}$ ciclo e ao nível do ensino em inglês nos $2 .^{\circ}$ e $3 .^{\circ}$ ciclos.

Apesar disto, os Vice-Reitores identificam um conjunto de iniciativas institucionais que, de alguma forma, encorajam o ensino-aprendizagem de línguas: criação de diversos cursos especialistas em línguas nos Departamentos e Faculdades de Línguas/Letras com integração de disciplinas de diversas línguas; criação de Centros de Línguas; oferta de cursos livres em línguas variadas nos Departamentos/ Faculdades /Centros de Línguas; oferta de cursos de Português como língua estrangeira para estudantes, professores e investigadores internacionais. Estas iniciativas são relacionadas, pelos Vice-Reitores, com as seguintes intenções: garantir diversidade nos campi; promover o contato intercultural; atrair estudantes estrangeiros e impulsionar a internacionalização das instituições.

No que concerne às crenças relativas ao ensino-aprendizagem de línguas no ES, quando questionados sobre a importância dessa aprendizagem por estudantes, professores e funcionários, os Vice-Reitores são bastante consonantes: acreditam que a 
aprendizagem de línguas por toda a comunidade académica é importante, enfatizando a aprendizagem do inglês e relacionando-a com a necessidade de internacionalização. Esta língua é, pois, percecionada como um instrumento que potencia a mobilidade dos diversos atores académicos. Apesar da preponderância dada ao inglês, todos os Vice-Reitores destacam o papel da língua portuguesa como estratégia de internacionalização do ES nacional, sublinhando o seu potencial na atração de estudantes, professores e investigadores da CPLP.

Para além disso, quatro dos Vice-Reitores sublinham o papel do espanhol e do francês no processo de comunicação e socialização dentro da academia, ou seja, na promoção do contato intercultural e na integração de estudantes, professores e investigadores de mobilidade, visto serem as línguas maternas e segundas línguas de milhões de falantes. Nessa linha, salientam a importância de dar às comunidades académicas a oportunidade de aprender outras línguas, contribuindo para o desenvolvimento da competência intercultural considerada uma mais-valia potenciadora da empregabilidade e da mobilidade, revelando uma perceção das línguas como trunfos económico-profissionais.

A análise revela, pois, uma certa tensão entre as práticas institucionais reportadas e as crenças dos Vice-Reitores: embora tenha havido uma diminuição dos cursos de línguas nos currículos e o inglês tenha adquirido o estatuto de língua franca na educação, os Vice-Reitores conferem importância à aprendizagem de línguas por toda a academia. Embora destaquem a aprendizagem do inglês, sublinham a importância de outras línguas como diferenciadoras económico-profissionais e facilitadoras interculturais.

\section{Línguas na investigação científica}

No que diz respeito à utilização das línguas na investigação científica, a análise revela tendências comuns. Com base no seu conhecimento relativo à publicação nas suas universidades, os Vice-Reitores afirmaram que a grande maioria dessa publicação é em inglês, enquanto o português é cada vez menos utilizado. Essa tendência levou as instituições a investir valores significativos na tradução de artigos para inglês, e a premiar investigadores que publicam em inglês em revistas indexadas, estratégias também reportadas em universidades escandinavas (Phillipson, 2017) e brasileiras (Fortes, 2016; Packer, 2016).

Estas práticas institucionais vão ao encontro das crenças dos Vice-Reitores sobre a utilização das línguas na investigação: sublinham que as línguas são, essencialmente, instrumentos que impulsionam a internacionalização da investigação e o sucesso científico-profissional dos investigadores e, nesse contexto, o inglês ocupa uma posição privilegiada. Neste sentido, e no que diz respeito à internacionalização da investigação, o inglês é um requisito fundamental para a disseminação e internacionalização 
do conhecimento científico, nomeadamente no que diz respeito à publicação. Esta posição detida pelo inglês é considerada pelos Vice-Reitores como imposição global, sentimento partilhado por atores de outras IES internacionais (Hultgren, Gregerson \& Thøgersern, 2014).

Apesar disto, os Vice-Reitores destacam a importância da utilização de outras línguas - português, espanhol e francês - na publicação, na constituição de redes de investigação e na organização de reuniões científicas, considerando-as línguas estratégicas para a internacionalização da investigação, a serem utilizadas de acordo com o domínio científico em questão e o impacto pretendido. No que concerne ao português, enfatizam a necessidade de lutar pelo seu reconhecimento como língua internacional da investigação, referindo-se ao valor económico de qualquer língua nacional e ao papel que pode desempenhar no desenvolvimento económico-social nacional. Nas suas vozes, isto implicará a criação de medidas concretas que promovam a indexação de publicações científicas escritas em português nas bases bibliométricas de referência. Para além disso, todos os Vice-Reitores acreditam que a existência crescente de grupos de investigação plurilingues oferece grandes benefícios no que diz respeito à relação entre línguas e internacionalização da investigação: publicação conjunta, construção de projetos conjuntos e o acesso subsequente a financiamento internacional.

A ênfase conferida à internacionalização da investigação e o papel que as línguas desempenham nesse processo estão, intimamente, relacionados com importância da internacionalização dos próprios investigadores e com o seu sucesso científico-profissional: todos os Vice-Reitores sublinham a importância do reconhecimento da comunidade científica internacional, que influi na avaliação institucional e individual. Assim, enfatizam que os investigadores portugueses, naturalmente preocupados com suas carreiras, são compelidos a publicar em inglês, sublinhando que a avaliação das unidades de investigação também leva em consideração a publicação em inglês pelos seus membros em revistas indexadas, a maioria das quais aceita textos exclusivamente nesta língua.

Deste modo, sobressai uma necessidade dialética de obter reconhecimento institucional e individual. De facto, as crenças dos Vice-Reitores mostram que os critérios para avaliação da investigação e dos investigadores influenciam fortemente as línguas escolhidas para publicação. Isto entra, de alguma forma, em conflito com a necessidade sentida pelos investigadores de divulgar seu trabalho em contexto nacional, sublinhada pelos Vice-Reitores, levando-os a enfatizar que os processos de avaliação da investigação em Portugal devem considerar publicações nacionais e internacionais, ponderadas de acordo com sua importância no campo em estudo.

Mais uma vez, esta análise evidencia uma tensão entre as práticas institucionais reportadas e as crenças dos Vice-Reitores: enquanto que ao nível das primeiras, se privilegia a utilização do inglês, primordial para a internacionalização das instituições e 
para o sucesso científico-profissional dos investigadores, ao nível das crenças, os Vice-Reitores enfatizam a necessidade de promover o português como língua de ciência.

\section{Desnvolvimento de políticas linguísticas institucionais de formação e de investigação}

No que concerne a práticas institucionais relativas ao desenvolvimento de PL de formação, os dados recolhidos mostram que nenhuma das universidades inquiridas possui estruturas institucionais responsáveis pela discussão e implementação de políticas linguísticas educativas. Apenas uma (U7) possui um grupo de trabalho, estabelecido pela Reitoria, que visa definir uma PL institucional a ser incluída no seu plano estratégico. Nenhuma tem recomendações institucionais ou diretrizes relativas a uma PL educativa e os Vice-Reitores afirmam não ter conhecimento da existência de discussão formal institucional sobre o assunto. Apesar disto, nas entrevistas, afirmam que existe um debate informal nas suas universidades entre professores nomeadamente quando há estudantes estrangeiros nas suas aulas.

Relativamente ao desenvolvimento de PL institucionais de investigação, dois Vice-Reitores reportam algumas iniciativas levadas a cabo nas suas universidades (U3 e U4): a existência de estruturas (comissões científicas das unidades de investigação) nas quais é discutida a questão das línguas utilizadas na publicação; a existência de documentos com resoluções sobre o uso das línguas na atividade científica, nomeadamente sublinhando a importância do inglês na publicação (planos estratégicos e relatórios de atividades); ações que estimulam a utilização de diversas línguas na atividade científica (seminários de investigação multilingues; prémios para investigadores que publicam em inglês e espanhol em revistas indexadas). No entanto, estas iniciativas só ocorrem em duas das universidades participantes neste estudo e centram-se na importância da língua inglesa na atividade científica.

Esta escassez de debate formal, estruturas e iniciativas relativas a PL para a formação e investigação colide com a importância conferida pelos Vice-Reitores ao desenvolvimento de PL institucionais explícitas. De facto, todos os inquiridos consideram esse desenvolvimento importante e estratégico por razões relacionadas com a internacionalização das instituições. Relativamente a PL educativas, salientam a importância de integrar a aprendizagem de línguas estrangeiras nos currículos, principalmente inglês, mas também a possibilidade de oferecer a estudantes, professores, investigadores e funcionários a oportunidade de aprender outras línguas através, por exemplo, da oferta de cursos livres. Além disso, enfatizam a necessidade de medidas que protejam o ensino em língua portuguesa, salientando que nem estudantes nem professores estão preparados para ensinar e aprender em inglês. Enfatizam, ainda, que o ensino em português permite atrair estudantes da CPLP. No que se refere a PL de investigação, os Vice-Reitores sublinham a necessidade de um equilíbrio entre inglês e português: a 
primeira para impulsionar a internacionalização ao nível global e a segunda para alcançar o público nacional e a CPLP.

É a partir destas crenças que os Vice-Reitores indicam algumas condições necessárias ao desenvolvimento de PL institucionais para a formação e para a investigação: - consciencialização de toda a comunidade académica para a relevância, quer para as instituições quer para as sociedades, de PL institucionais de teor multilingue;

- estabelecimento de espaços institucionais, reunindo estruturas de gestão, professores, investigadores, estudantes e funcionários que promovam uma discussão generalizada sobre PL no ensino superior;

- construção colaborativa dessas PL, tornando-as transversalmente compartilhadas e aceites, numa clara desvalorização de políticas top-down e valorizando a agência de diversos atores institucionais;

- integração das PL nas missões e planos estratégicos institucionais;

- apoio técnico e financeiro das agências governamentais.

De notar que estas condições têm vindo a ser apontadas como necessárias por estudos na área das PL no ensino superior em diferentes contextos (Ammon, 2006; Hamel, López \& Carvalhal, 2016).

A análise permite, uma vez mais, identificar uma tensão entre as práticas institucionais reportadas e as crenças dos Vice-Reitores: embora na maioria das universidades não exista debate formal, estruturas e iniciativas relativas a PL para a formação e investigação, os inquiridos acreditam que as suas instituições se devem preocupar com o desenvolvimento de PL institucionais, demonstrando uma consciencialização sobre a importância estratégica desse desenvolvimento.

\section{Das tensões à proposta de eixos de reflexão-ação para um multilinguismo estratégico no ES}

A análise dos dados permitiu identificar práticas institucionais e crenças convergentes nas universidades públicas que participaram no estudo. Para além disso, permitiu reconhecer uma tensão entre as práticas institucionais reportadas e as crenças dos Vice-Reitores. Efetivamente, e no que diz respeito ao ensino-aprendizagem de línguas e às línguas na investigação, enquanto as práticas respondem a um discurso global de teor mais económico-competitivo, com ênfase na importância do inglês na internacionalização da formação e da investigação, as crenças revelam-se próximas de um discurso mais plural, com ênfase na aprendizagem de línguas para além do inglês e na necessidade de promoção do português como língua de ciência. Também no que diz respeito ao desenvolvimento de PL institucionais de formação e de investigação se identifica uma tensão entre práticas e crenças: a quase inexistência de debate formal, 
estruturas e iniciativas relativas a PL institucionais em contradição com a crença geral de que as universidades as devem desenvolver.

Estes resultados permitem delinear algumas linhas de reflexão-ação dirigidas às IES que almejam o desenvolvimento de $\mathrm{PL}$ institucionais que conciliem um discurso de teor mais "económico-competitivo" com um discurso mais "plural", reconhecendo que não se tratam de discursos incompatíveis. Baseiam-se na possibilidade de um multilinguismo institucional estratégico, pragmático e complementar no âmbito do qual diversas línguas possam assumir funções diferenciadas consoante os contextos, os objetivos, o impacto desejado, o público-alvo e as necessidades individuais e institucionais. Vejamos, abaixo como se delineiam estas linhas à luz dos resultados do estudo em confronto com outros estudos em PL no ES.

\section{Locus institucional de discussão, reflexão e ação: consciencialização}

Torna-se necessário, antes de mais, que existam nas universidades espaços partiIhados, apoiados pelas estruturas de gestão, onde se potencie a discussão e reflexão sobre o papel das línguas na formação e na investigação (Pinto, 2012; Pinto \& Araújo e Sá, 2018). Estes espaços deverão agregar representantes dos diversos atores institucionais (professores, investigadores, estudantes, funcionários, órgão de gestão, ...) e indivíduos especialistas em questões de PL que, colaborativamente, poderão construir e disseminar uma visão partilhada acerca da relevância do desenvolvimento de PL de teor plurilingue. A discussão e reflexão partilhadas potenciarão uma ação mais forte e concertada que permitirá construir e disseminar pela academia argumentos (de teor social, cognitivo, económico, ambiental...) a favor da importância do investimento numa PL bottom-up que aposta na diversidade linguístico-cultural.

\section{Línguas e PL como "um assunto de toda a academia"}

O desenvolvimento de espaços de reflexão e de discussão heterogeneamente construídos e partilhados contribuirá para consciencializar os atores académicos acerca das mais-valias e potencialidades (educativas, cognitivas, sociais, económicas, ...) do plurilinguismo para as instituições e para os indivíduos. Trata-se de colocar a questão das línguas no ES como objeto de reflexão de todos os atores institucionais, levando-os a compreender que as línguas não são apenas instrumentos de comunicação e de internacionalização, mas que são, também, veículos de pensamento que garantem a pluralidade de perspetivas e que intervêm de forma estruturante na construção do conhecimento (Berthoud, 2015). Isto implica um diálogo académico entre especialistas na área das línguas e especialistas de outras disciplinas. 


\section{Plurilinguismo como estratégia menos óbvia de internacionalização}

Nas estratégias institucionais de internacionalização, a língua inglesa surge como condição sine qua non. Esta assunção relaciona-se com uma perspetiva redutora do conceito de internacionalização que, ao sobrevalorizar a língua inglesa, ignora os repertórios plurilingues dos atores académicos e as línguas locais, reforçando a existência de uma "monocultura epistemológica" que privilegia certas formas de conhecimento ignorando outras (Sousa Santos, 2014). Paradoxalmente, a internacionalização das IES que coloca a ênfase na mobilidade e na cooperação académica a diferentes níveis, não tem vindo a ser diretamente relacionada com a questão das línguas e com a diversidade linguístico-cultural que caracteriza as sociedades e as instituições e que é um motor de processos educativos e investigativos. Torna-se, pois, necessário destacar os benefícios adicionais do plurilinguismo como uma estratégia menos óbvia de internacionalização que permitirá, por exemplo, atrair estudantes internacionais falantes de língua portuguesa e de outras línguas para além do inglês, chegar a públicos diversos até agora não abrangidos pelos discursos monopolizadores da ciência (por exemplo, da Lusofonia) e estabelecer redes de formação e de investigação lusófonas e luso-hispânicas.

\section{Formação como impulsionadora do plurilinguismo}

Uma das mais prementes questões que, atualmente, se coloca à formação no ES é o desenvolvimento de competências transversais nos estudantes que potenciem a sua mobilidade, a capacidade para viverem em sociedades culturalmente diversas e a sua inserção num mercado de trabalho globalizado. É neste contexto que urge repensar os objetivos educativos e as competências a desenvolver. Neste âmbito, a integração de competências interculturais e plurilingues nos planos de estudo dos cursos e nos programas das unidades curriculares assume uma crucial importância, permitindo o desenvolvimento de uma consciencialização relativamente ao papel de mediação desempenhado pelas línguas. Desta forma, a formação poderá surgir enquanto impulsora do plurilinguismo e de PL de teor plurilingue ao nível de todas as missões universitárias (Coste, 2013; Pinto, 2012). Perspetivar estas linhas de reflexão-ação em termos práticos implica, fundamentalmente, agir sobre as crenças acerca das línguas e do plurilinguismo da comunidade académica (e da comunidade mais alargada) que se podem, de facto, consubstanciar como obstáculos ou impulsionadores à configuração e desenvolvimento de PL de teor mais ou menos multilingue. 


\section{Financiamento}

Este trabalho é financiado por Fundos Nacionais através da FCT - Fundação para a Ciência e a Tecnologia, I.P. no âmbito dos projetos SFRH/BPD/84560/2012 e UIDB/00194/2020.

\section{Referências Bibliográficas}

Ammon, U. (2006). Language planning for international scientific communication: an overview of questions and potential solutions. Current Issues in Language Planning, 7(1), 1-30.

Baldauf, R. (2006). Rearticulating the case for micro language planning in a language ecology context. Current Issues in Language Planning, 7(2-3), 147-170.

Berthoud, A.-C. (2015). The issues of multilingualism for the quality of higher education and research in the context of internationalisation. Disponivel em http://www.celelc.org/activities/Conferences/ Ressources/Ressources_Conference2015/Les-enjeux-du-multilinguisme Berthoud.pdf

Coste, D. (2013). Pluralité linguistique et transmission des savoirs. Quelques remarques. Synergies, 8 , 67-82.

Fortes, R. (2016). Política científica no Brasil: dilemas em torno da internacionalização e do inglês. Interfaces Brasil/Canadá, 16(1), 151-190.

Gallagher-Brett, A., \& Broady, E. (2012). Teaching languages in higher education. The Language Learning Journal, 40(3), 263-271.

Hamel, R., López, E., \& Carvalhal, T. (2016). Language policy and planning: challenges for Latin American universities. Current Issues in Language Planning, 17(3-4), 278-297.

Hultgren, K., Gregersen, F., \& Thøgersern, J. (2014). English in nordic universities: Ideologies and practices. Amsterdam: Benjamins.

Krippendorff, K. (2013). Content analysis. An introduction to its methodology. Los Angeles: Sage.

Liddicoat, A. (2016). Language planning in universities: Teaching, research and administration. Current Issues in Language Planning, 17(3-4), 231-241.

Ministério da Educação e Ciência. (2014). Uma estratégia para a internacionalização do Ensino Superior Português. Lisboa: MEC.

Miranda, N., Berdugo, M., \& Tejada, H. (2016). Conflicting views on language policy and planning at a Colombian university. Current Issues in Language Planning, 17(3-4), 422-440.

Packer, A. (2016). Cresce a adoção do inglês entre os periódicos SciELO do Brasil. Disponível em https:// blog.scielo.org/blog/2016/05/10/cresce-a-adocao-do-ingles-entre-os-periodicos-scielo-do-brasil

Phillipson, R. (2017). Myths and realities of 'global' English. Language Policy, 16(3), 313-331.

Pinto, S., \& Araújo e Sá, M. H. (em publicação). Scientific research and languages in Portuguese Higher Education Institutions. Language Problems and Language Planning.

Pinto, S., \& Araújo e Sá, M. H. (2019). Language education policy in Portuguese public universities: The voices of institutional stakeholders. Current Issues in Language Planning, 20(2), 140-159.

Pinto, S. (2012). As línguas na Universidade de Aveiro: discursos e práticas. (Tese de doutoramento). Universidade de Aveiro, Aveiro. Disponível em http://ria.ua.pt/bitstream/10773/8112/1/247487.pdf 
Pinto \& Sá: Políticas linguísticas de formação e de investigação em universidades públicas portuguesas

Siiner, M. (2016). University administrators as forced language policy agents. An institutional ethnography of parallel language strategy and practices at the University of Copenhagen. Current Issues in Language Planning, 17(3-4), 441-458.

Sousa Santos, B. (2014). Epistemologies of the south. Justice against epistemicide. Boulder: Paradigm Publishers.

Spolsky, B. (2004). Language policy. Cambridge: Cambridge University Press.

Zhao, S., \& Baldauf, R. (2012). Individual agency in language planning: Chinese script reform as a case study. Language Problems \& Language Planning, 36(1), 1-24.

\section{Susana Pinto}

Universidade de Aveiro, CIDTFF (Portugal)

Email: spinto@ua.pt

Orcid: https://orcid.org/0000-0001-7441-4140

Maria Helena Araújo e Sá

Universidade de Aveiro, CIDTFF (Portugal)

Email: helenasa@ua.pt

Orcid: https://orcid.org/0000-0002-6623-9642

Correspondência

Susana Pinto

Universidade de Aveiro, Departamento de Educação e Psicologia

Campus Universitário de Santiago, 3810-193 Aveiro

Data de submissão: Março 2019

Data de avaliação: Maio 2019

Data de publicação: Abril 2020 